\title{
Topic Introduction
}

\section{Nucleic Acid Platform Technologies}

\author{
Oliver Rando
}

Every microarray experiment is based on a common format. First, a large number of nucleotide "spots" are arrayed onto a substrate, typically a glass slide, a silicon chip, or microbeads. Second, a complex population of nucleic acids (isolated from cells, selected from in vitro-synthesized libraries, or obtained from another source) is labeled, typically with fluorescent dyes. Third, the labeled nucleic acids are allowed to hybridize to their complementary spot(s) on the microarray. Fourth, the hybridized microarray is washed, allowing the amount of hybridized label to then be quantified. Analysis of the raw data generates a readout of the levels of each species of RNA in the original complex population. This introduction includes several examples of microarray applications and provides a discussion of the basic steps of most microarray experiments.

Microarrays can be run in "one-color" or "two-color" formats. In a one-color microarray, a single sample (e.g., RNA from liver cells) is labeled, and the abundance of a species of RNA is inferred from the intensity of the spot(s) complementary to the relevant gene. Because, for each spot, hybridization is affected by a panoply of factors, interpretation of single-color experiments can be complicated. Practically, however, many of these complicating factors are resolvable by using the proper bioinformatics tools during data analysis. Two-color microarrays (Fig. 1) use a competitive hybridization in which one nucleic acid sample is labeled with one color (green), and a related sample is labeled with a second color (red). Following hybridization and removal of unbound nucleic acid, the microarray is scanned with lasers to detect where the red- and green-labeled molecules have bound. The intensity of each spot is determined, and the red/green ratio is measured for each spot. During data analysis, this ratio can be used to measure the ratio of the amount of related nucleic acid molecules in the two samples. For example, if RNA from normal liver is tagged green and RNA from a liver tumor is labeled red, then red spots would represent RNAs that are up-regulated in the tumor, and green spots would represent down-regulated RNAs.

Tiling microarrays consist of regularly spaced oligonucleotides that provide dense coverage of a genome or portion of a genome. For example, yeast chromosome III was tiled using oligonucleotides 50 -nt long spaced every 20 bases; that is, spot 1 comprised bases $1-50$, spot 2 bases $21-70$, and so forth. Tiling microarrays therefore interrogate continuously along a chromosomal path, enabling applications, such as transcript structure and protein localization, to be performed with almost complete genomic coverage. One disadvantage to tiling microarrays is that not all genomic sequence is equally suited to microarray applications. For example, not all 50-mers in a genome are unique, leading to gaps in the tiling path where hybridization-based approaches cannot determine whether the hybridizing material comes from copy 1 or copy 2 (or copy $N$ ) in the genome. Furthermore,

From the Molecular Cloning collection, edited by Michael R. Green and Joseph Sambrook.

(C) 2019 Cold Spring Harbor Laboratory Press

Cite this introduction as Cold Spring Harb Protoc; doi:10.1101/pdb.top096388 
O. Rando

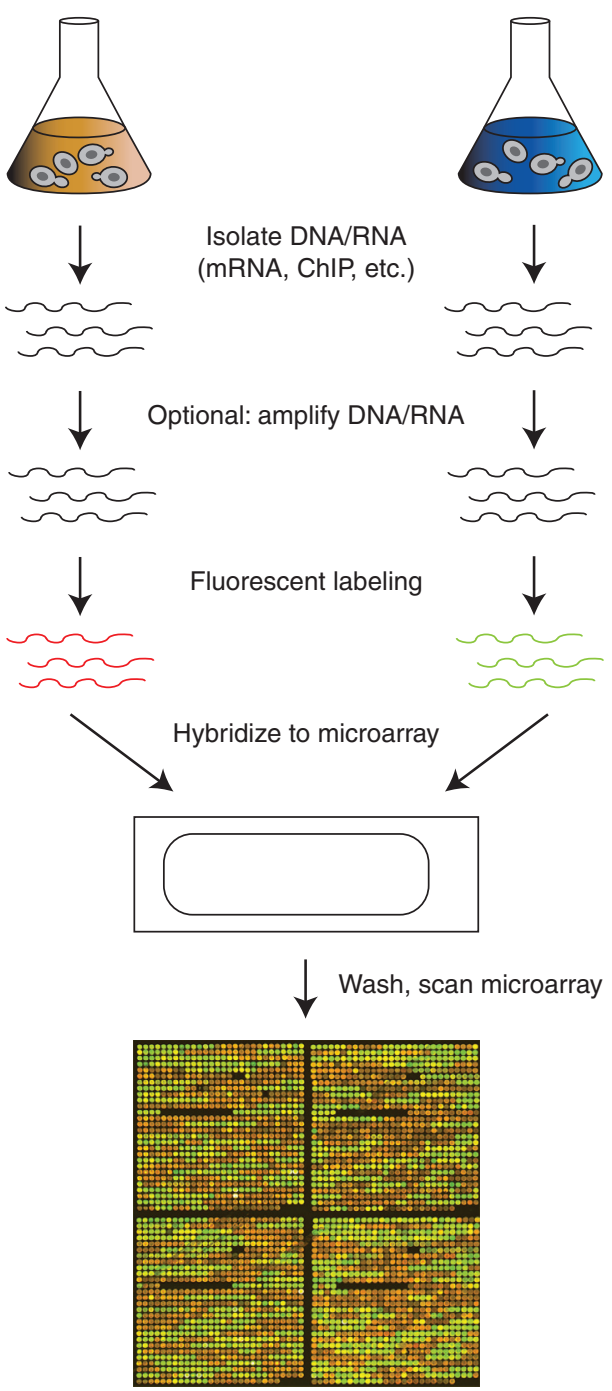

FIGURE 1. Basic steps in setting up and running a two-color microarray experiment. The details of each step are explained in the text.

hybridization-related properties such as the percentage of $\mathrm{A}$ and $\mathrm{T}$ residues and oligonucleotide melting temperature vary from target to target, although many issues like these can be resolved by normalizing two-color arrays. Normalization, which compensates for systematic technical differences between spots and/or arrays, clarifies the systematic biological differences between samples.

\section{MICROARRAY APPLICATIONS}

Although the majority of early microarray studies focused on mRNA expression profiling, microarrays can be used for any purpose that involves comparison of two nucleotide populations-for example, comparison of RNAs extracted from normal and tumor cells. In this section, we list several examples (by no means exhaustive) of published microarray analyses.

mRNA Expression Levels

Early gene expression microarrays consisted of arrayed spots each containing a cDNA corresponding to a single gene. For example, one of the first yeast gene expression microarrays consisted of about 6000 spots composed of cDNA corresponding to nearly every gene in the Saccharomyces cerevisiae genome (DeRisi et al. 1997). cDNA microarrays have fallen into disuse because of the advent of 
oligonucleotide microarrays. Advances in oligonucleotide synthesis make it possible to generate gene expression microarrays composed of long oligonucleotides that have been designed to be complementary to a given gene. Oligonucleotides can be synthesized and sold in soluble form, which are then arrayed by a spotting robot, as with cDNA arrays. Alternatively, several commercial microarray suppliers use "in situ" oligonucleotide synthesis, in which oligonucleotides are printed directly onto the microarray substrate. This process allows for much more flexible generation of oligonucleotide microarrays and, thus, is used more often than soluble oligonucleotide arrays because a laboratory need not purchase an excess of oligonucleotides to run microarray experiments.

\section{Monitoring Changes in the Transcriptome}

A typical mRNA expression experiment begins with the isolation of poly $(\mathrm{A})^{+} \mathrm{RNA}$ from two related populations of cells, such as yeast growing at $30^{\circ} \mathrm{C}$ and yeast that have been shifted from $30^{\circ} \mathrm{C}$ to $37^{\circ} \mathrm{C}$. Fluorophore-labeled cDNA is prepared from each mRNA population. Typically, the cyanine dyes used are $\mathrm{Cy} 5$ (pseudocolored red in microarray images) and $\mathrm{Cy} 3$ (pseudocolored green). The labeled cDNAs are mixed and hybridized to a microarray. After hybridization and washing, the microarray is scanned with lasers that excite the fluorophores, and the image is processed, generating a $\mathrm{Cy} 5 / \mathrm{Cy} 3$ ratio for every spot on the array. The microarray data are normalized, and a list of the $\mathrm{Cy} 5 / \mathrm{Cy} 3$ ratios per gene is generated, which can be analyzed for genome-wide changes in the transcriptome.

The microarray data are normalized so that the average $\log _{2}(\mathrm{Cy} 5 / \mathrm{Cy} 3)$ is 0 ; this is a necessary step because the absolute intensity of either channel (Cy5 or Cy3) is subject to a wide range of modifying influences, such as input RNA level and labeling efficiency, among others. This normalization should always be kept in mind in interpreting microarray studies. For example, microarray results for a comparison between liver RNA and sperm RNA populations might indicate that a number of RNAs are relatively enriched in sperm, but the absolute numbers of those RNAs per nucleus are still probably lower in sperm than in liver given the disparity in RNA abundance in the two types of cells.

mRNA Abundance

The experiment described above is capable of providing information about the relative abundances of every mRNA expressed in the two cell populations. What about absolute expression levels? These can be assayed in two ways. First, single-color experiments using the Affymetrix platform provide a reasonable measure of mRNA abundance-the intensity of a given spot is related to the absolute abundance of its complementary mRNA in the original labeled population. Second, for two-color experiments, a straightforward way to estimate transcript abundance is to compare labeled mRNA with labeled genomic DNA, which, in principle, should be present in a single copy per gene (unless the gene in question comes from a multicopy family). Thus, gDNA is labeled with Cy3, mRNA is labeled with $\mathrm{Cy} 5$, and $\mathrm{Cy} 5 / \mathrm{Cy} 3$ ratios provide a relative measure of mRNA abundance. These ratios can be converted to absolute mRNA abundance if the absolute abundance of any RNA is already known. Normalization can be used to set the absolute level of these RNAs, and all remaining RNA abundances can be inferred from their $\mathrm{Cy} 5 / \mathrm{Cy} 3$ ratios relative to the $\mathrm{Cy} 5 / \mathrm{Cy} 3$ ratios for the known standards.

\section{Comparative Genomic Hybridization}

Comparative genomic hybridization (CGH) is used to detect changes in DNA copy number across a genome (Pollack et al. 1999). In a CGH experiment, the two populations of genomic DNA are fluorescently labeled using Klenow DNA polymerase. Labeled DNA is compared using the same experimental design as for analysis of mRNA expression. Changes in copy number are reflected in the $\mathrm{Cy} 5 / \mathrm{Cy} 3$ ratios. This method has been used most commonly in the study of tumors, where there is a well-documented role both for gene loss and for amplification in the process of oncogenesis. By analyzing changes in gene copy number, regional amplification or even whole chromosome copy-number changes have been characterized. Variants of this approach have also been used to 
O. Rando

characterize replication timing, as DNA isolated during S phase shows variations in copy number, based on whether or not the segment of genomic DNA has replicated.

\section{Determination of Transcript Structure}

Although changes in mRNA levels can be analyzed easily using microarrays constructed with one probe (spot) per gene, different methods are required to detect variations in transcript structure. These variations include changes in splicing patterns and in the start and stop sites for transcription. These and other changes in transcript structure can be detected and mapped using tiling microarrays. For example, transcriptional start sites can be mapped to $\sim 10$ - to 20 -nt resolution by a variant of the absolute abundance hybridization method described above. Briefly, mRNA is labeled with Cy5 and hybridized against Cy3-labeled genomic DNA. In principle, an expressed RNA should appear as a long "square wave" of high Cy5/Cy3 ratios: $\mathrm{Cy} 5 / \mathrm{Cy} 3$ ratios should be low in spots corresponding to genomic sequences that flank those complementary to the RNA. High Cy5/Cy3 ratios should start at spots containing sequences corresponding to the $5^{\prime}$ end of the RNA and should continue to the end of the RNA (Fig. 2). Indeed, this technique has been used to determine transcriptional start sites to $\sim 20$-nt resolution in yeast (Yuan et al. 2005) and to detect and map novel transcripts in human cells (Shoemaker et al. 2001).

Variations in splicing patterns can also be detected and mapped using microarrays. With tiling arrays, expressed exons should appear as peaks of Cy5/Cy3. Another way to determine exon inclusion is to use an exon microarray, in which each spot on the array corresponds to a single exon. When probing an exon microarray, for a given tissue all of a gene's expressed exons will show high $\mathrm{Cy} 5 / \mathrm{Cy} 3$ (Shoemaker et al. 2001).

Tiling microarrays have also been used to discover noncoding RNAs and novel genes. The reason for this is obvious - a classical, expression microarray only covers what is known, whereas a tiling microarray can be designed to contain an entire genome. For example, tiling microarrays of

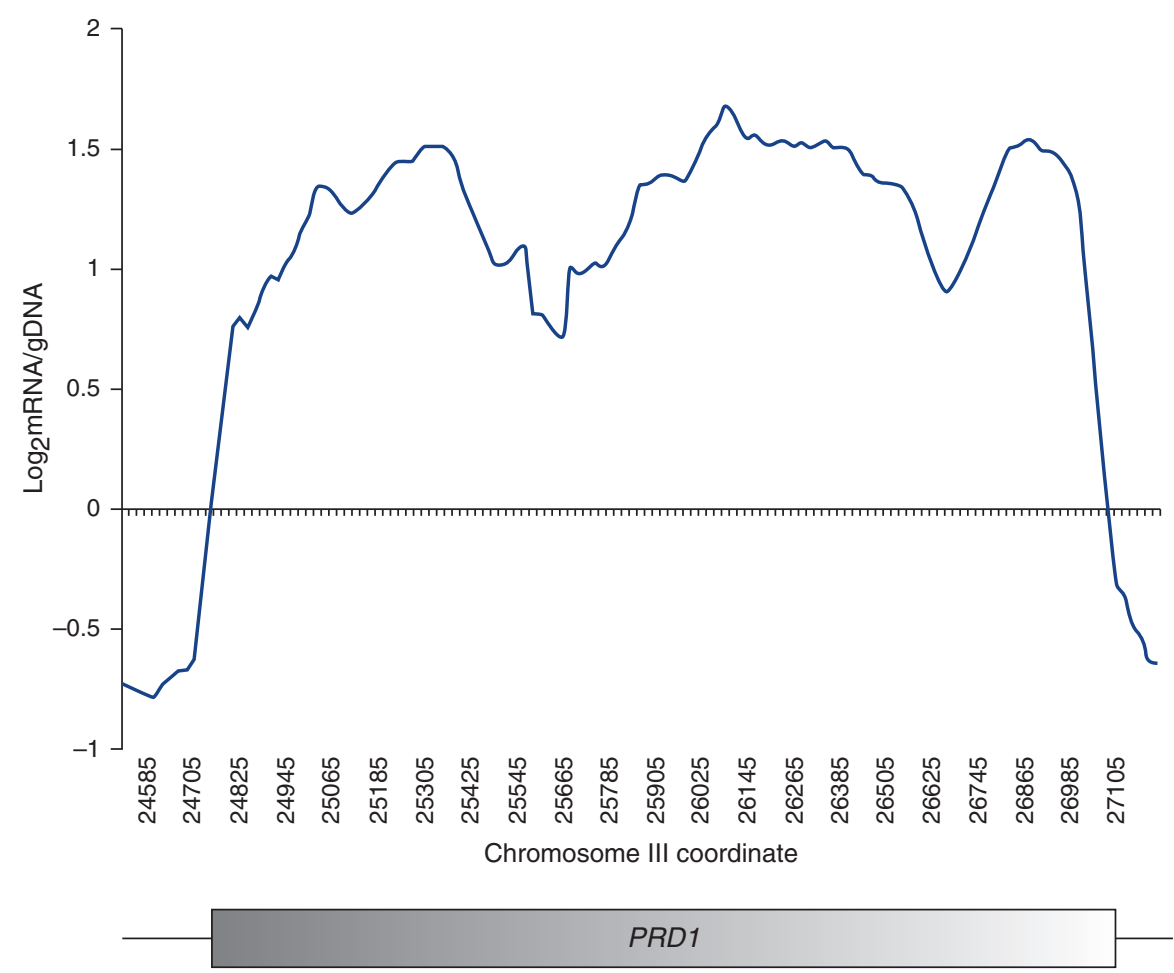

FIGURE 2. Tiling array analysis of mRNA structure. Shown are data from a tiling array hybridization of labeled mRNA versus labeled genomic DNA, with the $y$-axis showing $\log _{2}$ of the ratio mRNA/gDNA, and the $x$-axis showing the chromosomal coordinates of microarray probes. 
chromosomes 21 and 22 revealed the nearly pervasive low-level transcription of the human genome (Kapranov et al. 2002).

Identifying RNA-Protein Interactions

RNA molecules associated with specific RNA-binding proteins have been identified using immunoprecipitation assays to isolate a protein of interest. The associated RNA molecules (or regions of RNA) are then compared with nonspecific RNAs that have been isolated in control immunoprecipitations lacking antibody (Hieronymus and Silver 2003; Gerber et al. 2004).

Subcellular Localization of RNA Populations

The functional properties of RNAs (e.g., translation) can be assayed by microarray using more sophisticated fractionation techniques. In an early study, polysomal RNAs were analyzed by microarray techniques (Arava et al. 2003). Subcellular localization of RNAs can also be studied when appropriate purification techniques exist; for example, isolation of dendrites from neurons has been used to identify the relevant RNA populations (Eberwine et al. 2002).

\section{Protein Localization Studies}

At present, one of the most popular applications of tiling microarrays is known as "chromatin immunoprecipitation on chip" (or ChIP-on-chip). By this method, the location of a specific protein, such as a transcription factor, is assayed through the use of chromatin immunoprecipitation. Briefly, proteins are covalently cross-linked to the genome using formaldehyde, and chromatin is sheared by sonication to small ( $\sim 500 \mathrm{bp})$ fragments. Using an antibody specific to a given protein (with either an antibody to an epitope tag, a protein-specific antibody, or even an antibody against a specific modification state), DNA associated with the protein in question is isolated by immunoprecipitation. After washing, the cross-links are dismantled and genomic DNA is isolated. The DNA is typically amplified, and the amplified material is labeled and hybridized competitively against labeled amplification reactions from no-antibody controls or from pre-IP material. Analysis of ChIP-on-chip microarray results provides a snapshot of a transcription factor's location on the genome and has proven to be a tremendously powerful method for studying transcriptional regulation by transcription factors and chromatin (Ren et al. 2000; Iyer et al. 2001).

Nuclease Accessibility as a Structural Probe

Tiling microarrays can also be hybridized with genomic DNA that has been treated diagnostically with a nuclease before labeling the DNA. This method takes advantage of the fact that chromatin packaging of the genome dramatically affects nuclease accessibility. Two nucleases have been used in genomewide studies. First, DNase I-hypersensitive sites have long been known to occur at genomic regulatory elements such as promoters, enhancers, and insulators. Here, chromatin is lightly digested with DNase I, sites of cleavage are isolated, and DNA surrounding cleavage sites is interrogated by tiling microarray analysis (Sabo et al. 2006). Second, micrococcal nuclease (MNase) is a nonprocessive nuclease with preference for the linker DNA between nucleosomes. Analysis of the DNA protected from MNase cleavage including comparison to whole-genomic DNA has proven to be an invaluable tool in genome-wide nucleosome mapping studies (Yuan et al. 2005).

\section{Splicing Microarrays}

Although exon microarrays (see above) can monitor expression of each exon represented on the array, the structure of the transcript cannot be discerned. Specifically, exon connectivity is not detected using exon microarrays. For example, expression of exons 1, 2, 3, and 5 could indicate a single species containing all four exons, or two or more distinct species (1-2-5 and 1-3-5, etc.). To determine connectivity of exons requires the use of splicing microarrays, which are comprised of oligonucleotides complementary to specific splice junctions. 
O. Rando

\section{Resequencing and SNP Detection}

The sequence specificity of DNA hybridization has also been leveraged for genome analyses such as "resequencing" and polymorphism detection. In these applications, each genomic location is represented on the microarray by several oligonucleotides that differ by a single nucleotide. In other words, if the genomic region of interest has the sequence AATGCCA, oligonucleotides containing AATTCCA, AATCCCA, and AATACCA will also be printed. Hybridization of genomic DNA to these oligonucleotides can be used to determine mutations or sequence polymorphisms at the site interrogated by the set of oligonucleotides.

One way to use microarrays for resequencing is the so-called sequence capture protocol (Hodges et al. 2007). A microarray is printed with oligonucleotides corresponding to genomic regions of interest. DNA or RNA is hybridized to the microarray, and all complementary sequences are retained on the microarray through the washing protocols. After retention of desired genomic regions via hybridization, this hybridized material is eluted and sequenced using high-depth sequencing methods.

\section{PERFORMING MICROARRAY EXPERIMENTS}

Although the specific bound oligonucleotides and labeled targets and details of the analysis of microarray hybridizations differ depending on the experimental questions being asked, most microarray experiments involve six steps.

1. Design a microarray.

2. Print or purchase a microarray.

3. Isolate and amplify the DNA or RNA target material.

4. Label the DNA or RNA with fluorescent groups.

5. Hybridize the labeled targets to the microarray.

6. Analyze the microarray hybridization results.

For the remainder of the introduction, we discuss each of these steps in turn. Protocols are provided for printing a microarray in-house (Protocol: Printing Microarrays [Rando 2019a]) and for amplifying DNA and RNA following isolation (Protocol: Round A/Round B Amplification of DNA for Microarrays [Rando 2019b], Protocol: T7 Linear Amplification of DNA (TLAD) for Microarrays [Rando 2019c] and Protocol: Amplification of RNA for Microarrays [Rando 2019d]). Several techniques for adding fluorescent moieties to the nucleic acids are provided (Protocol: Direct Cyanine-dUTP Labeling of RNA for Microarrays [Rando 2019e], Protocol: Indirect AminoallyldUTP Labeling of RNA for Microarrays [Rando 2019f], Protocol: Direct Cyanine-dCTP Labeling of DNA for Microarrays [Rando 2019g] and Protocol: Indirect Labeling of DNA for Microarrays [Rando 2019h]). Protocol: Blocking Polylysines on Homemade Microarrays (Rando 2019i) explains how to block the positive charges of the polylysines bound to homemade microarrays. Finally, there is a detailed generalized protocol for hybridization of labeled nucleic acids to a microarray, as well as scanning, formatting, and storing the microarray hybridization data (Protocol: Hybridization to Homemade Microarrays [Rando 2019j]). A brief guide to microarray analysis is contained in Introduction: Analysis of Microarray and RNA-seq Expression Profiling Data (Hung and Weng 2017a) and described in Protocol: Analyzing Microarray Data (Hung and Weng 2017b).

\section{Designing a Microarray}

A great variety of microarrays are commercially available, and for most researchers, an off-the-shelf product will suffice. If not, customized microarrays can be ordered from several manufacturers or designed (and printed) in-house. The appropriate design of a microarray will depend on the application for which it is to be used. There are, however, some basic design principles that are common to most applications. We consider two types of basic microarray: the gene expression microarray and the 
tiling microarray. Specialized formats, such as splicing arrays and resequencing arrays, have been designed for different organisms, and we point interested readers to the relevant literature (Hacia 1999; Mockler et al. 2005; Blencowe 2006; Hughes et al. 2006; Calarco et al. 2007; Cowell and Hawthorn 2007; Gresham et al. 2008).

\section{Gene Expression Microarrays}

Designing oligonucleotides for microarrays requires expertise in bioinformatics, but some basic design properties are easily understood. First, choose an appropriate oligonucleotide length. The majority of oligonucleotide microarrays today are printed with oligonucleotides 50-70 nt long. Designing oligonucleotides of optimal lengths requires consideration of several factors, including signal strength, specificity, cost, and efficiency of synthesis. Shorter oligonucleotides are more likely to crosshybridize to many different regions of a given genome and will often have very low melting temperatures, making hybridization technically problematic. A detailed analysis of oligonucleotide length versus sensitivity and specificity can be found in Hughes et al. (2001). A reasonable rule of thumb is that for most applications, 60-nt oligonucleotides provide the best balance between these competing constraints.

The second consideration in oligonucleotide design is the degree of specificity or complementarity between the oligonucleotides on the microarray and the RNA species in the organism of interest. In general, the oligonucleotides should be designed so that they are complementary to only one RNA species. Commonly, BLAST searches are used to screen the sequence of each oligonucleotide against the relevant genomic sequence to identify the potential for multiple hits in the genome of interest. Ideally, rather than focusing on a cut-off BLAST value, the user should select for each gene the oligonucleotide having the lowest-scoring second match in the genome.

The third design consideration is optimization of the hybridization properties. Several features can be optimized, but the most important is the melting temperature $\left(T_{\mathrm{m}}\right)$ of the oligonucleotide. Specifically, the $T_{\mathrm{m}} \mathrm{s}$ of all of the oligonucleotides on the array should be within as narrow a window as is feasible. Other characteristics, such as entropy (i.e., the complexity of the sequence), GC\%, and selfcomplementarity should be optimized as well. Software tools are available to help with oligonucleotide design, ranging from publicly available tools like ArrayOligoSelector (see below) to commercial services provided by microarray companies.

As an example, consider a case in which an investigator wishes to design gene expression microarrays for a nonmodel organism with a recently sequenced genome. The first step in designing gene expression microarrays is, of course, to identify the genes. As this is routinely performed in any genome sequencing effort, we will assume that genes have already been predicted using standard tools (Zhang 2002; Ashurst and Collins 2003; Brent 2005; Solovyev et al. 2006).

\section{ArrayOligoSelector}

Once coding regions are identified, oligonucleotides need to be designed for each gene. Many programs exist for oligonucleotide selection, including ArrayOligoSelector (Bozdech et al. 2003a,b), a commonly used program that is freely available at http://arrayoligosel.sourceforge.net/.

ArrayOligoSelector is designed to analyze a complete genome and prepare oligonucleotides of a user-defined length. For every oligonucleotide, ArrayOligoSelector calculates scores for uniqueness, sequence complexity, self-annealing, and GC content. Uniqueness is a measure of the theoretical difference in binding energy between a given oligonucleotide and either its perfect match or the next most homologous genomic sequence. Sequence complexity allows the user to filter oligonucleotides with homopolymeric tracts, which otherwise may cause hybridization problems. The self-annealing score is a measure of the secondary structure generated by the self-annealing of an oligonucleotide. Self-annealing is another potential source of hybridization problems. Finally, it is important to minimize variation in GC percentage among the oligonucleotide sequences, both to minimize $T_{\mathrm{m}}$ variation and to minimize variability in the fluorescence intensity among the spots. 
O. Rando

When running ArrayOligoSelector, the following features can be specified: oligonucleotide length, GC\%, number of oligonucleotides per gene, sequences to mask, and uniqueness cutoff. Common oligonucleotide lengths are 60 -mers or 70 -mers. GC\% will vary depending on the GC\% of the genome in question and will typically be chosen as the genomic coding region average. The number of oligonucleotides per gene will depend on whether the microarray is to be purchased or printed inhouse. If oligonucleotides are purchased for in-house printing, then cost and the printable spot density will preclude using more than one or two oligonucleotides per gene. Alternatively, if commercial arrays are to be used, then the number of spots available will determine the number of oligonucleotides to be chosen per gene. Masking sequences are not commonly specified, but if a problematic short repeat element is present in the genome, then it is sometimes valuable to mask it out of the microarray oligonucleotides. The uniqueness cutoff is typically left blank, which will result in the default value being used (for additional information, see the ArrayOligoSelector manual).

The output of ArrayOligoSelector can be filtered by the experimenter. For example, it is often desirable to use oligonucleotides located toward the $3^{\prime}$ ends of genes because reverse-transcriptasebased labeling is more efficient in this region.

\section{Tiling Microarrays}

Oligonucleotide design for tiling microarrays is more straightforward than for gene expression microarrays because tiling presumes that essentially all of the oligonucleotides within a region of interest will be included on the microarray. The simplest tiling design involves choosing nucleotides 1-50 (say) of some region to be tiled as spot 1, nucleotides 21-70 as spot 2, and so forth. Once all of the oligonucleotides have been designed, BLAST can be used to find oligonucleotides with multiple identical matches in the genome of interest, and these oligonucleotides can be removed. More subtle tiling designs incorporate a small amount of "wiggle" in the oligonucleotide location; thus spot 2 might run from nucleotides 16-65, spot 3 might run from 45-94, and so forth. By doing this, the process of matching hybridization properties, such as $T_{\mathrm{m}}$ and GC\%, is better than with a simple tiling microarray.

\section{Printing or Purchasing a Microarray}

Once oligonucleotides have been designed, microarrays can be printed commercially. Alternatively, oligonucleotides can be synthesized commercially or by an in-house core facility, and then printed inhouse using a spotting robot (see Protocol: Printing Microarrays [Rando 2019a]).

\section{Isolating and Amplifying Nucleic Acid Samples for Hybridization to Microarrays}

Most of the sample preparation procedures for microarrays follow standard protocols, many of which can be found elsewhere in this manual. For example, comparative genomic hybridization (CGH) analyses use genomic DNA isolated from samples of interest. Gene expression or splicing studies use either total RNA or mRNA. Protein localization analysis starts with material isolated via chromatin immunoprecipitation (ChIP). Typically, however, the intended assays for many of these protocols are based on blotting techniques or quantitative PCR readouts, which often require only nanograms of material. Microarray labeling, on the other hand, typically requires several micrograms of nucleic acid; therefore, an amplification step is necessary before labeling.

\section{General Notes on Amplification}

Because amplification of the nucleic acid that will be used to generate labeled microarray targets occurs before the hybridization step, it must not bias representation of any particular sequences in the genome. Thus, unbiased (or minimally biased) whole-genome amplification protocols are a key component of many microarray applications.

We have provided three amplification protocols: two for DNA (Protocol: Round A/Round B Amplification of DNA for Microarrays [Rando 2019b] and Protocol: T7 Linear Amplification of 
DNA (TLAD) for Microarrays [Rando 2019c]) and one for RNA (Protocol: Amplification of RNA for Microarrays [Rando 2019d]). There are several practical items to keep in mind while performing an amplification protocol. First, when trying an amplification method for the first time, start with a large and easily obtainable pool of material (e.g., liver RNA) and amplify an aliquot of the original pool. Microarray comparisons between the amplified material and the original "bulk" pool then provide a valuable readout of amplification biases. A perfect amplification would result in a "yellow" array with no spots showing any differences between the bulk and amplified material. Second, avoid contaminating your sample with anything that might contain DNA or RNA. Even tiny amounts of foreign nucleic acids will be amplified, contaminating your sample and corrupting your microarray experiments. Always wear gloves and use filter pipette tips when performing the isolation and amplification protocols. Finally, always include a control amplification with water only (no DNA or RNA) to ensure that the reagents are not contaminated with amplifiable material.

\section{RNA Amplification for Expression Profiling}

Less common than DNA amplification, RNA amplification is nonetheless required in experiments that use small populations of cells, such as may occur in neurobiological studies using laser-captured cell populations. RNA amplification kits are available from several vendors (e.g., Ambion), or amplification can be performed as described in Protocol: Amplification of RNA for Microarrays (Rando 2019d).

\section{Generating Fluorescently Labeled Nucleic Acid Targets}

Labeling nucleic acids for use in microarrays is similar for most microarray platforms, except for Affymetrix microarrays. For most platforms (homemade or commercial), fluorescent molecules are attached to DNA by the Klenow fragment of DNA polymerase I. For labeling RNA, reverse transcriptase is used to prepare labeled cDNA. Labeling methods can include a fluorescent dNTP in the labeling reaction (Protocol: Direct Cyanine-dUTP Labeling of RNA for Microarrays [Rando 2019e] and Protocol: Direct Cyanine-dCTP Labeling of DNA for Microarrays [Rando 2019g]). Because this approach is rapid but expensive, a cheaper but lengthier alternative first incorporates aminoallyl nucleotides into the nucleic acid molecules and then couples the aminoallyl group to the fluorophore (Protocol: Indirect Aminoallyl-dUTP Labeling of RNA for Microarrays [Rando 2019f] and Protocol: Indirect Labeling of DNA for Microarrays [Rando 2019h]). In all of the labeling protocols, the source nucleic acids can be either unamplified or amplified before labeling.

Hybridizing to a Microarray

Methods for hybridization of labeled materials to a microarray differ significantly when using homeprinted microarrays versus commercial microarrays. For home-printed microarrays, slides must first be blocked because polylysines remaining on the slide will cause significant background binding to labeled material unless they are neutralized by reaction with succinic anhydride. Protocol: Blocking Polylysines on Homemade Microarrays (Rando 2019i) and Protocol: Hybridization to Homemade Microarrays (Rando 2019j) provide methods, respectively, for blocking and hybridizing to homemade arrays. When using commercial microarrays, hybridization protocols are typically provided. Following hybridization, microarrays are scanned in a benchtop scanner. The data are collected, formatted, and stored digitally for subsequent analysis.

\section{Analyzing Microarray Data}

The tools used for analysis of microarray data will depend on the experimental question being asked: Localization studies require a different set of tools than do gene expression studies. Some of the available resources are described in Introduction: Analysis of Microarray and RNA-seq Expression Profiling Data (Hung and Weng 2017a) and Protocol: Analyzing Microarray Data (Hung and Weng 2017b). Here, we outline some of the basic steps in data analysis. 
The first step in microarray data analysis is to remove bad data (i.e., data from spots that were "flagged" because they were obscured by fluorescent precipitate, etc.), and to normalize the remaining data. Working with a .gpr file, which is the standard output of GenePix software, we typically eliminate all flagged targets and then work exclusively with the $\log _{2}$ ratio data. More advanced users may consider using specific features, such as Foreground and Background intensities.

Most two-color microarray studies are normalized to an average $\log _{2}$ ratio value of 0 . The assumption implicit in this normalization is that there was no overall change in whatever is being measured. Thus, it is important to remember when looking at such normalized data that relative values are being measured, not absolute values. Practically, normalization can be performed by averaging the unflagged log ratio value, then subtracting this value from every one of the entries in the column. This can be done by hand in spreadsheet programs or using common commands in languages such as MATLAB, Perl, or R.

Once normalization is completed, the list can be sorted by log ratio value to identify genes that are dramatically up-regulated or down-regulated (if gene expression) or identify loci with high levels of enrichment of the protein in question. At this point, data analysis paths will diverge depending on the questions being asked. However, because many microarray studies involve multiple microarrays, it is often useful to cluster data to identify genes or loci that share similar behavior.

For clustering and visualization, numerous programs are available online. We use the classic Cluster and TreeView programs (Eisen et al. 1998), available online at SourceForge (http://sourceforge.net/) or via the Eisen laboratory website (http://www.eisenlab.org/). Because sample files that guide the formatting of files for clustering are also available, formatting will not be described here. Briefly, however, data will be loaded into Cluster, various thresholds will be set (fraction of data missing for a given gene, number of genes changing over some threshold, etc.), and one of several clustering algorithms will be used. The output of the clustering can be visualized with TreeView, allowing users to generate the classic "heatmap" view of their microarray data.

\section{ACKNOWLEDGMENTS}

The protocols introduced here were modified from protocols written and generously provided by Ash Alizadeh, Chih Long Liu, Audrey Gasch, Jason Lieb, Bing Ren, and L. Ryan Baugh.

\section{REFERENCES}

Arava Y, Wang Y, Storey JD, Liu CL, Brown PO, Herschlag D. 2003. Genome-wide analysis of mRNA translation profiles in Saccharomyces cerevisiae. Proc Natl Acad Sci 100: 3889-3894.

Ashurst JL, Collins JE. 2003. Gene annotation: Prediction and testing. Annu Rev Genomics Hum Genet 4: 69-88.

Blencowe BJ. 2006. Alternative splicing: New insights from global analyses. Cell 126: 37-47.

Bozdech Z, Llinas M, Pulliam BL, Wong ED, Zhu J, DeRisi JL. 2003a. The transcriptome of the intraerythrocytic developmental cycle of Plasmodium falciparum. PLoS Biol 1: e5. doi: 10.1371/journal.pbio.0000005.

Bozdech Z, Zhu J, Joachimiak MP, Cohen FE, Pulliam B, DeRisi JL. 2003 b. Expression profiling of the schizont and trophozoite stages of Plasmodium falciparum with a long-oligonucleotide microarray. Genome Biol 4: R9. doi: 10.1186/gb-2003-4-2-r9.

Brent MR. 2005. Genome annotation past, present, and future: How to define an ORF at each locus. Genome Res 15: 1777-1786.

Calarco JA, Saltzman AL, Ip JY, Blencowe BJ. 2007. Technologies for the global discovery and analysis of alternative splicing. Adv Exp Med Biol 623: 64-84.

Cowell JK, Hawthorn L. 2007. The application of microarray technology to the analysis of the cancer genome. Curr Mol Med 7: 103-120.

DeRisi JL, Iyer VR, Brown PO. 1997. Exploring the metabolic and genetic control of gene expression on a genomic scale. Science 278: 680-686.
Eberwine J, Belt B, Kacharmina JE, Miyashiro K. 2002. Analysis of subcellularly localized mRNAs using in situ hybridization, mRNA amplification, and expression profiling. Neurochem Res 27: 1065-1077.

Eisen MB, Spellman PT, Brown PO, Botstein D. 1998. Cluster analysis and display of genome-wide expression patterns. Proc Natl Acad Sci 95: 14863-14868.

Gerber AP, Herschlag D, Brown PO. 2004. Extensive association of functionally and cytotopically related mRNAs with Puf family RNAbinding proteins in yeast. PLoS Biol 2: e79. doi: 10.1371/journal .pbio.0020079.

Gresham D, Dunham MJ, Botstein D. 2008. Comparing whole genomes using DNA microarrays. Nat Rev Genet 9: 291-302.

Hacia JG. 1999. Resequencing and mutational analysis using oligonucleotide microarrays. Nat Genet 21: 42-47.

Hieronymus H, Silver PA. 2003. Genome-wide analysis of RNA-protein interactions illustrates specificity of the mRNA export machinery. Nat Genet 33: 155-161.

Hodges E, Xuan Z, Balija V, Kramer M, Molla MN, Smith SW, Middle CM, Rodesch MJ, Albert TJ, Hannon GJ, McCombie WR. 2007. Genomewide in situ exon capture for selective resequencing. Nat Genet 39: $1522-1527$.

Hughes TR, Mao M, Jones AR, Burchard J, Marton MJ, Shannon KW, Lefkowitz SM, Ziman M, Schelter JM, Meyer MR, et al. 2001. Expres- 
sion profiling using microarrays fabricated by an ink-jet oligonucleotide synthesizer. Nat Biotechnol 19: 342-347.

Hughes TR, Hiley SL, Saltzman AL, Babak T, Blencowe BJ. 2006. Microarray analysis of RNA processing and modification. Methods Enzymol 410: $300-316$.

Hung J-H, Weng Z. 2017a. Analysis of microarray and RNA-seq expression profiling data. Cold Spring Harb Protoc doi: 10.1101/pdb .top093104.

Hung J-H, Weng Z. 2017b. Analyzing microarray data. Cold Spring Harb Protoc doi: 10.1101/pdb.prot093112.

Iyer VR, Horak CE, Scafe CS, Botstein D, Snyder M, Brown PO. 2001. Genomic binding sites of the yeast cell-cycle transcription factors SBF and MBF. Nature 409: 533-538.

Kapranov P, Cawley SE, Drenkow J, Bekiranov S, Strausberg RL, Fodor SP, Gingeras TR. 2002. Large-scale transcriptional activity in chromosomes 21 and 22. Science 296: 916-919.

Mockler TC, Chan S, Sundaresan A, Chen H, Jacobsen SE, Ecker JR. 2005. Applications of DNA tiling arrays for whole-genome analysis. Genomics 85: $1-15$

Pollack JR, Perou CM, Alizadeh AA, Eisen MB, Pergamenschikov A, Williams CF, Jeffrey SS, Botstein D, Brown PO. 1999. Genome-wide analysis of DNA copy-number changes using cDNA microarrays. Nat Genet 23: 41-46.

Rando O. 2019a. Printing microarrays. Cold Spring Harb Protoc doi: 10.1101/ pdb.prot096396.

Rando O. 2019b. Round A/Round B amplification of DNA for microarrays. Cold Spring Harb Protoc doi: 10.1101/pdb.prot096404.

Rando O. 2019c. T7 linear amplification of DNA (TLAD) for microarrays. Cold Spring Harb Protoc doi: 10.1101/pdb.prot096412.

Rando O. 2019d. Amplification of RNA for microarrays. Cold Spring Harb Protoc doi: 10.1101/pdb.prot096420.
Rando O. 2019e. Direct cyanine-dUTP labeling of RNA for microarrays. Cold Spring Harb Protoc doi: 10.1101/pdb.prot096438.

Rando O. 2019f. Indirect aminoallyl-dUTP labeling of RNA for microarrays. Cold Spring Harb Protoc doi: 10.1101/pdb.prot096446.

Rando O. 2019g. Direct cyanine-dCTP labeling of DNA for microarrays. Cold Spring Harb Protoc doi: 10.1101/pdb.prot096453.

Rando O. 2019h. Indirect labeling of DNA for microarrays. Cold Spring Harb Protoc doi: 10.1101/pdb.prot096461.

Rando O. 2019i. Blocking polylysines on homemade microarrays. Cold Spring Harb Protoc doi: 10.1101/pdb.prot096479.

Rando O. 2019j. Hybridization to homemade microarrays. Cold Spring Harb Protoc doi: 10.1101/pdb.prot096487.

Ren B, Robert F, Wyrick JJ, Aparicio O, Jennings EG, Simon I, Zeitlinger J, Schreiber J, Hannett N, Kanin E, et al. 2000. Genome-wide location and function of DNA binding proteins. Science 290: 2306-2309.

Sabo PJ, Kuehn MS, Thurman R, Johnson BE, Johnson EM, Cao H, Yu M, Rosenzweig E, Goldy J, Haydock A, et al. 2006. Genome-scale mapping of DNase I sensitivity in vivo using tiling DNA microarrays. Nat Methods 3: 511-518.

Shoemaker DD, Schadt EE, Armour CD, He YD, Garrett-Engele P, McDonagh PD, Loerch PM, Leonardson A, Lum PY, Cavet G, et al. 2001. Experimental annotation of the human genome using microarray technology. Nature 409: 922-927.

Solovyev V, Kosarev P, Seledsov I, Vorobyev D. 2006. Automatic annotation of eukaryotic genes, pseudogenes and promoters. Genome Biol 7: S10.1-S10.12.

Yuan GC, Liu YJ, Dion MF, Slack MD, Wu LF, Altschuler SJ, Rando OJ. 2005. Genome-scale identification of nucleosome positions in S. cerevisiae. Science 309: 626-630.

Zhang MQ. 2002. Computational prediction of eukaryotic protein-coding genes. Nat Rev Genet 3: 698-709. 


\section{Nucleic Acid Platform Technologies}

Oliver Rando

Cold Spring Harb Protoc; doi: 10.1101/pdb.top096388 originally published online June 5, 2019

\begin{tabular}{cc}
$\begin{array}{c}\text { Email Alerting } \\
\text { Service }\end{array}$ & Receive free email alerts when new articles cite this article - click here. \\
\hline $\begin{array}{c}\text { Subject } \\
\text { Categories }\end{array}$ & Browse articles on similar topics from Cold Spring Harbor Protocols. \\
& Analysis of Microarrays (19 articles) \\
& Analysis of Microarrays, general (13 articles) \\
& Bioinformatics/Genomics, general (192 articles) \\
& Expression Analysis of RNA (14 articles) \\
& Genomic Analysis (10 articles) \\
& High-Throughput Analysis, general (155 articles) \\
& Microarrays (54 articles) \\
& Microarrays, general (55 articles) \\
& Spotted Glass Microarrays (14 articles) \\
& Target Preparation (13 articles) \\
\hline
\end{tabular}

